\title{
Immunity in Rhodnius prolixus: Trypanosomatid-vector Interactions
}

\author{
P Azambuja $/{ }^{+}$, D Feder, CB Mello*, SAO Gomes, ES Garcia \\ Departamento de Bioquímica e Biologia Molecular, Instituto Oswaldo Cruz, Av. Brasil 4365, 21045-900 Rio de \\ Janeiro, RJ, Brasil *Departamento de Biologia Geral, Universidade Federal Fluminense, Niterói, RJ, Brasil
}

Key words: immunity - Rhodnius prolixus - vector-interactions

Many insects respond to a bacterial infection with the stimulation of distinct cellular and humoral defense system, that cooperate in a more or less integrated way to decrease the chance of microorganisms becoming pathogens. Cellular reactions include phagocytosis, nodule formation, and in some cases encapsulation and other factors related to immune system as prophenoloxidase (proPO) system, lectin, lysozyme and induced peptides such cecropin, attacin and other factors (Ratcliffe \& Rowley 1979, Dunn 1986, Boman \& Hultmark 1987). Feder et al. (1997) demonstrated, when Rhodnius prolixus was challenged with Enterobater cloacae, the importance of the effects of diet components on the immune reactivity. For example, plasma diet induced immune depression. Ecdysone therapy counteracted the immune depression in Rhodnius larvae fed on plasma diet alone (Feder et al. 1997).

In spite of the extensive research conducted over the last few years on the molecular bases of these responses, the regulation on the blood sucking insect defense reaction against parasite remains relatively poor understood. Many trypanosomatides develop their cycles in the hemocel and/ or digestive tract of the insect vector. While in $R$. prolixus the development of Trypanosoma cruzi (causative agent of Chagas diesease) is confined to the gut lumen, $T$. rangeli develops in the gut but clearly invades the hemolymph and survives free

\footnotetext{
This work was supported by grants from Conselho Nacional de Desenvolvimento Científico e Tecnológico (to PA and ESG), Fundação Oswaldo Cruz (Papes project to PA), Escola Brasil-Argentina de Biotecnologia do Ministério de Ciência e Tecnologia e Projeto, Programa Brasil-Alemanha (Probal) da Capes, Ministério da Educação (to ESG), and Padct. PA, ESG, SAOG, CBM are CNPq, and DF Faperj/Fiocruz research fellows.

${ }^{+}$Corresponding author. Fax: +55-21- 590.3495. E-mail: azambuja@gene.dbbm.fiocruz.br

Received 9 June 1999

Accepted 9 August 1999
}

in the blood or inside the hemocytes (Brener 1972, D’Alessandro 1976, Garcia \& Azambuja 1991).

The importance of the vector immune system as an essential component of the parasite-insect vector relationship has recently been recognized (Molyneux et al. 1986, Kaaya 1989, Ingram \& Molyneux 1991, Mello et al. 1995). In this paper we present findings to support the hypothesis that the vector immune system may have a role in the trypanosomatid-triatomine interaction.

\section{TRIATOMINE REACTIONS AGAINST T. CRUZI}

Isola et al. (1981, 1986) suggested the involvement of factors from the intestine in the development of T. cruzi. The parasite develops within the digestive tube of the vector where the presence of digestive enzymes and a hemolytic factor produce a potentially hostile environment for this parasite (Azambuja et al. 1983, Garcia 1987, 1989a,b). Recently, Mello et al. (1995) studied the course of infection of T. cruzi (clone Dm28c) and its interaction with hemolymph components of $R$. prolixus. The main conclusion of this paper was that T. cruzi had no division, did not induce trypanolytic and antibacterial molecules, but induced high lysozyme and nodule formation levels when inoculated in the hemolymph. Furthermore, the number of $T$. cruzi in the hemolymph was directly correlated with PO activities which decreased soon after the parasite disappear. It seems that $T$. cruzi has no ability to escape from some immune reactions of the hemolymph.

Mello et al. (1996) also made a differential in vivo and in vitro study of three strains of $T$. cruzi in the gut and hemolymph of $R$. prolixus. Basically, they demonstrated that both T. cruzi strains Dm28c and $\mathrm{Cl}$ successfully infected the gut as evidenced by over than $50 \%$ of insects having infective forms in feces and urine a month after feeding with parasites. Concomitantly, both of these strains were agglutinated but no lysed by the crop homogenates. By contrary, T. cruzi Y strain, showed no agglutination, but some lysis in the crop, and consequently rapidly disappeared from the gut, as already described by Azambuja et al. (1989 a,b). After in- 
oculating into the hemocel, only the $\mathrm{Cl}$ strain survived and maintained with high number of parasite circulating. That strain was also the only one which agglutinated in the hemolymph. The other two strains, Dm28c and Y, rapidly disappeared from the hemocel probably due to the action of the cellular immune reaction. Finally, these author demonstrated that the carbohydrate on the parasite surfaces had differences between the three strains. Therefore, gut and hemolymph lectins and parasite surface carbohydrates could be important determinants of infectivity in the trypanosometriatomine interaction. It is difficulty of making generalizations about such parasite-host interactions without taking into consideration many strains of parasites tested. The activity of agglutinins found in the gut tissues and hemolymph of Rhodnius was also tested using rabbit erythrocytes (Ratcliffe et al. 1996). They demonstrated that crop, midgut, hindgut, and crude hemolymph contained hemagglutinins. The agglutinins were produced by the vector rather than absorbed and concentrated from the blood meal. The gut extracts obtained from insects fed on rabbit plasma had strong activity despite the fact that rabbit plasma failed to agglutinate the erythrocytes. Carbohydrate and glycoprotein inhibition studies of the crop, midgut, and hindgut also failed to detect a simple sugar which inhibited the agglutination of these tissues. The only inhibitory compounds for any of the gut hemagglutinins or crop parasite agglutinin were sugars linked to p-nitrophenol such as pnitrophenyl- $\alpha$-D-galactopyranoside which effectively inhibited all the agglutinins. Also, pnitrophenol without linkage to any sugar residue was also inhibitory. Inhibition studies with the crude hemolymph agglutinin demonstrated, in agreement with Pereira et al. (1981), that galactose containing sugars, including galactose, lactose, D-galactosamine, and methyl-D-galactopyranoside were inhibitory. An SDS-PAGE gel of the hemolymph lectin following elution from the galactose-bound, Sepharose 6B minicollumn, demonstrated a single band of the lectin with molecular weight of $40 \mathrm{kDa}$.

\section{INTERACTION BETWEEN T. RANGELI AND R. PROLIXUS}

As we already described, $T$. rangeli develops in the gut, invades and survives in the hemolymph and thus it is probably recognized by the immune system of the insect vector. Tobie $(1968,1970)$, Takle (1988) and Mello et al. (1995) demonstrated that following inoculation into the hemolymph of Rhodnius, $T$. rangeli survives and multiplies. In vitro experiment of $R$. prolixus hemolymph from insects inoculated with $T$. rangeli, there were nei- ther consistent change in erythrocyte agglutination nor induction of antitrypanosome activity. However, levels of PO, lysozyme, and hemocyte numbers in the hemolymph significantly enhanced after inoculation of this parasite. Nodule formation increased during the entire experiment indicating that $T$. rangeli although recognized and enclosed within the cellular defenses, is capable to survive and to utilize the cells involved for multiplication (Takle 1988).

Pereira et al. (1981) and Gregorio and Ratcliiffe (1991b) described lectins in the crop, midgut, and hemolymph of $R$. prolixus able to agglutinate trypanosomes. Gregorio a Ratcliffe (1991b) also reported a wider distribution of lectins in T. infestans, than in Rhodnius, and postulated that these molecules may have imparted to Triatoma into refractoriness to infect with $T$. rangeli. An alternative candidate for immune modulation of $T$. rangeli invasion is the proPO. The proPO, an inactive precursor of $\mathrm{PO}$ found in the plasma fraction of hemolymph (Pye 1974) or in the hemocytes (Leonard et al. 1985) of invertebrates, can be activated by proteases, such as trypsin and chymotrypsin, bacteria, and fungi, or by their cell wall components (Ashida et al. 1983). In $R$. prolixus, the activation of the proPO pathway was observed in insects inoculated with bacteria or trypanosomatids (Azambuja et al. 1986, 1989a,b, Gregorio \& Ratcliffe 1991b, Mello et al. 1995, Feder et al. 1997). Gregorio and Ratcliffe (1991a), using in vitro activation of the proPO system, showed that the $T$. rangeli infection of $R$. prolixus, at least in part, may have been related to the suppression of the activation of proPO in the presence of the parasite. Mello et al. (1995) demonstrated that the rate of $T$. rangeli development in the hemolymph of $R$. prolixus linearly enhanced within four days of infection. Numerous short epimastigotes of $T$. rangeli were present until day two in the hemolymph but after this time, they disappear to be replaced by a massive colonization by long epimastigotes. Recently, basing on these findings, Gomes et al. (1999) studied the in vivo and in vitro activation of the proPO in $R$. prolixus infected with short and long epimastigotes of $T$. rangeli, separately. The in vitro activation of the proPO pathway was low in the absence of fat body, hemolymph, and both parasites. A higher PO activity was observed when short, but not long, epimastigotes of $T$. rangeli were incubated with hemolymph, fat body, and the substrate L-DOPA. Similarly, the PO activity of hemolymph taken from infected insects with long epimastigotes showed a low activity if compared with hemolymph obtained from insects inoculated with short parasites. Based on these results, Gomes et al. (1999) suggest that 
(a) factor(s) in the hemolymph as well as in the fat body may be released by the presence of short epimastigotes of $T$. rangeli and which results in the activation of the proPO system in $R$. prolixus. Gomes et al. (data not published) demonstrated that the factor(s) must be proteases detected in the hemolymph of insects which were fed on, or inoculated with, short epimastigotes of $T$. rangeli. In this case they were not observed in the fat body. No protease activiy could be observed in both hemolymph and fat body taken from insects inoculated with, or fed on, long epimastigotes. In support of these findings Mello et al. (1999) demonstrated that in $R$. prolixus hemocyte monolayers, $T$. rangeli is able of inducing hemocyte/parasite clump formation. They also observed that purified hemolymph galactoside-binding lectin markedly increased the formation of clump by $T$. rangeli in $R$. prolixus hemocyte monolayers, consequently with an enhance in clump size and hemocyte aggregation. This enhancement of nodule formation was specifically inhibited by addition of the galactose ligant for the lectin. Furthermore, pure lectin affected the motility and survival of short epimastigotes, but no the long ones, when they were incubated in vitro. Based on the present findings, we postulate that differential activation of the proPO pathway and the cellular reaction induced by short and long epimastigotes of $T$. rangeli is responsible for differences in the development and establishment of the parasites in the hemolymph of $R$. prolixus. Thus, we delineate, for the first time, an in vivo mechanism related to immune reactions that is involved in the infection of $T$. rangeli in the vector.

\section{REFERENCES}

Ashida M, Ishizaki Y, Iwahana H 1983. Activation of prophenoloxidase by bacterial cell walls or b-1,3glucans in plasma of the silkworm, Bombyx mori. Biochem Biophys Res Commun 113: 562-568.

Azambuja P, Freitas CC, Garcia ES 1986. Evidence and partial characterization of an inducible antibacterial factor in the hemolymph of Rhodnius prolixus. J Insect Physiol 32: 807-812.

Azambuja P, Guimarães JA, Garcia ES 1983. Haemolytic factor from the crop of Rhodnius prolixus: evidence and characterization. J Insect Physiol 29: 833-839.

Azambuja P, Mello CB, Garcia ES 1989a. Immunity to Rhodnius prolixus: inducible peptides against bacteria and trypanosomes, p. 270-276. In D Borovsky \& A Spielman (eds), Host Regulated Developmental Mechanisms in Vector Arthropods, Vero Beach, Florida.

Azambuja P, Mello CB, D’Escoffier LN, Garcia ES 1989 b. In vitro cytotoxicity of Rhodnius prolixus hemolytic factor and mellitin towards trypanosomatids. Brazilian J Med Biol Res 22: 597599.
Boman HG, Hultmark D 1987. Cell free immunity in insects. Annu Rev Microbiol 41: 103 - 126.

Brener Z 1972. A new aspect of Trypanosoma cruzi life cycle in the invertebrate host. J Protozool 19: 2327.

D’Alessandro A 1976. Biology of Trypanosoma (Herpetosoma) rangeli, p. 327-403. In WHR Lumsden, DA Evans (eds), Biology of Kinetoplastida, Vol. 1, Academic Press, London.

Dunn PE 1986. Biochemical aspects of insect immunology. Annu Rev Entomol 31: 321- 339.

Feder D, Mello CB, Garcia ES, Azambuja P 1997. Immune reactions in Rhodnius prolixus: influence of nutrition and ecdysone. J Insect Physiol 43: 513-519.

Garcia ES 1987. The digestion of Triatominae, p. 4758. In RR Brenner \& AM Stoka (eds), Chagas' Disease Vectors, Vol. II, CRC Press Inc., Florida.

Garcia ES, Azambuja P 1991. Development and interactions of Trypanosoma cruzi in insect vector. Parasitol Today 7: 240-244.

Gomes SAO, Feder D, Thomas NES, Garcia, ES, Azambuja P 1999. Rhodnius prolixus infected with Trypanosoma rangeli: in vivo and in vitro experiments. J Invertebr Pathol 73: 289-293.

Gregorio EA, Ratcliffe NA 1991a. The prophenoloxidase system in vitro interaction of Trypanosoma rangeli with Rhodnius prolixus and Triatoma infestans haemolymph. Parasite Immunol 13: 551-564.

Gregorio EA, Ratcliffe NA 1991b. The distribution of agglutinins and lytic activity against Trypanosoma rangeli and erythrocytes in Rhodnius prolixus and Triatoma infestans tissue extracts and haemolymph. Mem Inst Oswaldo Cruz 86: 181-186.

Ingram GA, Molyneux DH 1991. Insect lectins: role in parasite-vector interactions, p. 103-127. In DD Kilpatrick, E van Dreissche \& TC Hansen (eds), Lectin Reviews 1, Sigma Chemical Co., St Louis.

Isola EID, Lammel EM, Katzin AM, Gonzlez Cappa SM 1981. Influence of organ extracts of Triatoma infestans on the differentiation of Trypanosoma cruzi. J Parasitol 67: 53-58

Isola ELD, Lammel EM, Gonzalez Cappa SM 1986. Trypanosoma cruzi: Differentiation after interaction of epimastigotes and Triatoma infestans intestinal homogenate. Exp Parasitol 62: 329-335.

Kaaya GP 1989. A review of the progress in recent years on research and understanding of immunity in insect vectors of human and animal diseases. Insect Sci Appl 10: 751-769.

Leonard C, Soderhall K, Ratcliffe NA 1985. Studies on prophenoloxidase and protease activity of Blaberus craniifer haemocytes. J Insect Biochem 15: 803-810.

Mello CB, Azambuja P, Garcia ES, Ratcliffe NA 1996. Differential in vitro and in vivo behavior of three strains of Trypanosoma cruzi in the gut and hemolymph of Rhodnius prolixus. Exp Parasitol 82: 112-121.

Mello CB, Garcia ES, Ratcliffe NA, Azambuja P 1995. Trypanosoma cruzi and Trypanosoma rangeli: Interplay with hemolymph components of Rhodnius prolixus. J Invertebr Pathol 65: 261-268. 
Mello CB, Nigan Y, Garcia ES, Azambuja P, Newton RP, Ratcliffe NA 1999. Studies in a haemolymph lectin isolated from Rhodnius prolixus and it's interaction with Trypanosoma rangeli. Exp Parasitol 91: 289-296.

Molyneux DH, Takle G, Ibrahim EA, Ingram GA 1986. Insect immunity in trypanosomatidae, p. 117-144. In AM Lackie, Immune Mechanisms in Invertebrate Vectors, Clarendon Press, Oxford.

Pye AE 1974. Microbial activation of prophenoloxidase from immune insect larvae. Nature 251: 610-612.

Pereira MEA, Andrade AFB, Ribeiro JMC 1981. Lectins of distinct specificity in Rhodnius prolixus interact selectively with Trypanosoma cruzi. Science 211: 597-600.

Ratcliffe NA, Rowley AF 1979. Role of hemocytes in defense against biological agents, p. 331-414. In AP
Gupta, Insect Hemocytes: Development, Forms, Functions and Techniques, New York: Cambridge University Press.

Ratcliffe NA, Nigam Y, Mello CB, Garcia ES, Azambuja P 1996. Trypanosoma cruzi and erythrocyte agglutinins: a comparative study of occurance and properties in the gut and hemolymph of Rhodnius prolixus. Exp Parasitol 83: 83-93.

Takle GB 1988. Studies on the cellular immune response of insects toward the insect pathogen Trypanosoma rangeli. J Invertebr Pathol 51: 64-72.

Tobie EJ 1968. Fate of some culture flagellates in the haemocoel of Rhodnius prolixus. J Parasitol 54: 1040-1046.

Tobie EJ 1970. Observations on the development of Trypanosome rangeli in the haemocoel of Rhodnius prolixus. J Invertebr Pathol 15: 118-125. 